\title{
A highly accurate solenoid valve driver with current sensing circuits for brake systems
}

\author{
Chang-woo Lee ${ }^{1,2}$ and Oh-kyong Kwon ${ }^{2 a)}$ \\ ${ }^{1}$ Mando Global R\&D Center, \\ 619 Sampyung-dong, Bundang-gu, Seongnam-si, Gyeonggi-do, Korea \\ 2 Department of Electronics Engineering, Hanyang University, \\ Seoul 133-791, Korea
}

a)okwon@hanyang.ac.kr

\begin{abstract}
In this paper, we propose a highly accurate solenoid valve driver with high- and low-side current sensing circuits. The proposed solenoid valve driver improves the current accuracy by sensing the current of the solenoid coil alternately in the on- and off-states of the power MOS transistor. In the on-state, the low-side sensing circuit senses the current flowing through the low-side transistor. In the off-state, the high-side sensing circuit senses the current flowing through the high-side transistor. The proposed solenoid valve driver was implemented using a $0.11 \mu \mathrm{m}$ BCDMOS process. The measurement results show that the proposed solenoid valve driver achieves a high current accuracy of less than $\pm 4 \%$ over the entire current range from $0 \mathrm{~A}$ to $2.25 \mathrm{~A}$.
\end{abstract}

Keywords: solenoid valve driver, current sensing, ESC, ABS, ECU, BCDMOS

Classification: Power devices and circuits

\section{References}

[1] K. Kobayashi and H. Inoue: U.S. Patent 6634724 (2003).

[2] H. E. Tseng, et al.: "The development of vehicle stability control at ford," IEEE/ASME Trans. Mechatron. 4 (1999) 223 (DOI: 10.1109/3516.789681).

[3] K. Reif: Automotive Mechatronics (Springer, Wiesbaden, 2015) 355.

[4] Infineon Technology: TLE8242 datasheet (2010) http://www.infineon.com.

[5] STmicroelectronics: L9352B datasheet (2013) http://www.st.com.

[6] L. Piato, et al.: "A flexible approach for inductive load driver in automotive application," 2015 12th International Workshop on Intelligent Solutions in Embedded Systems (WISES) (2015) 97.

[7] M. Branciforte, et al.: "ANN and non-integer order modeling of ABS solenoid valves,” IEEE Trans. Contr. Syst. Technol. 19 (2011) 628 (DOI: 10.1109/ TCST.2010.2049999).

[8] H. Jung, et al.: "Resistance estimation of a PWM-driven Solenoid," Int. J. Automotive Technol. 8 (2007) 249 (DOI: 10.1.1.159.8548).

[9] H. Jung, et al.: "Robust solenoid current control for EHB," SAE International (2005) (DOI: 10.4271/2005-01-1583). 
[10] Y. Ren, et al.: "Analytical loss model of power MOSFET," IEEE Trans. Power Electron. 21 (2006) 310 (DOI: 10.1109/TPEL.2005.869743).

[11] STmicroelectronics: AN604 application note (2011) http://www.st.com.

[12] STmicroelectronics: STPS3150U datasheet (2016) http://www.st.com.

[13] H. P. Forghani-zadeh and G. A. Rincon-Mora: "Current-sensing techniques for DC-DC converters," The 2002 45th Midwest Symposium on Circuits and Systems 2 (2002) 577 (DOI: 10.1109/MWSCAS.2002.1186927).

\section{Introduction}

Recently, automobile brake systems have been evolving from the anti-lock brake system (ABS) and electronic stability control (ESC) system to the integrated dynamic brake (IDB) system and iBooster system [1]. The ABS and ESC systems have been mass-produced in many countries and have contributed to reducing traffic accidents [2]. Next generation brake systems, such as IDB and iBooster, eliminate the need for a brake booster, thereby reducing both the system weight and load on the engine to improve the efficiency of an automobile. These systems generally consist of a 3-phase motor for driving a master cylinder, solenoid valves for controlling the operation of the conventional brake system (CBS) and ESC system, a hydraulic control unit (HCU) with a hydraulic path, and an electronic control unit (ECU). The ECU consists of a microcontroller unit (MCU), driver ICs, and a control unit having the safety functions necessary for controlling these components. In particular, the solenoid valves in the brake system precisely control the brake pressure when the brake system operates with other systems, such as vehicle radar and vision cameras. In addition, the smart cruise control system assisted by the vehicle radar controls the solenoid coil with several tens of $\mathrm{mA}$ current.

Fig. 1 shows the typical HCU circuit of the ESC system [3], which includes 12 solenoid valves. The IDB and iBooster systems have the same (or greater) number of valves as the ESC system, which are necessary to realize the conventional brake and ESC systems without a mechanical booster. Among these 12 valves, 8 valves are used to perform pulse width modulation (PWM) control for high and low pressure operation, and 4 valves are used to open and close the hydraulic path.

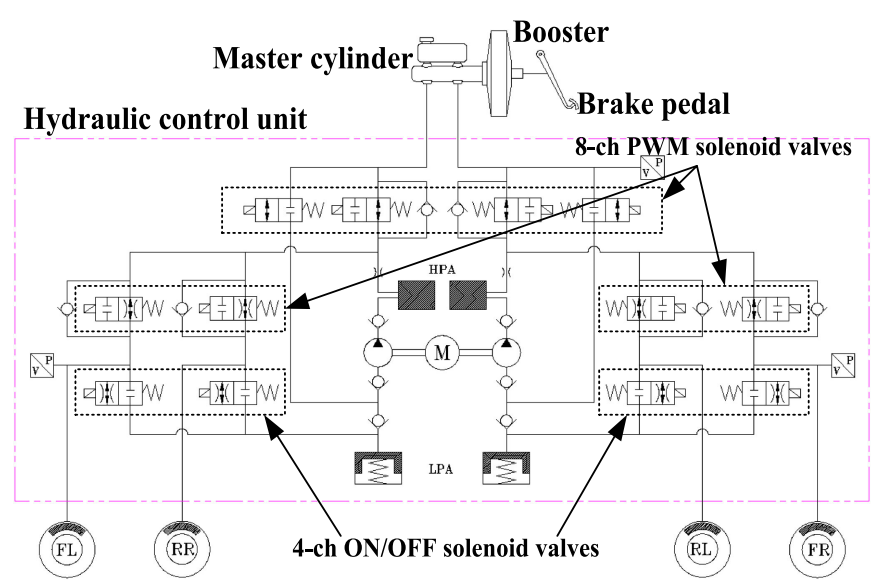

Fig. 1. Typical HCU circuit of the ESC system. 


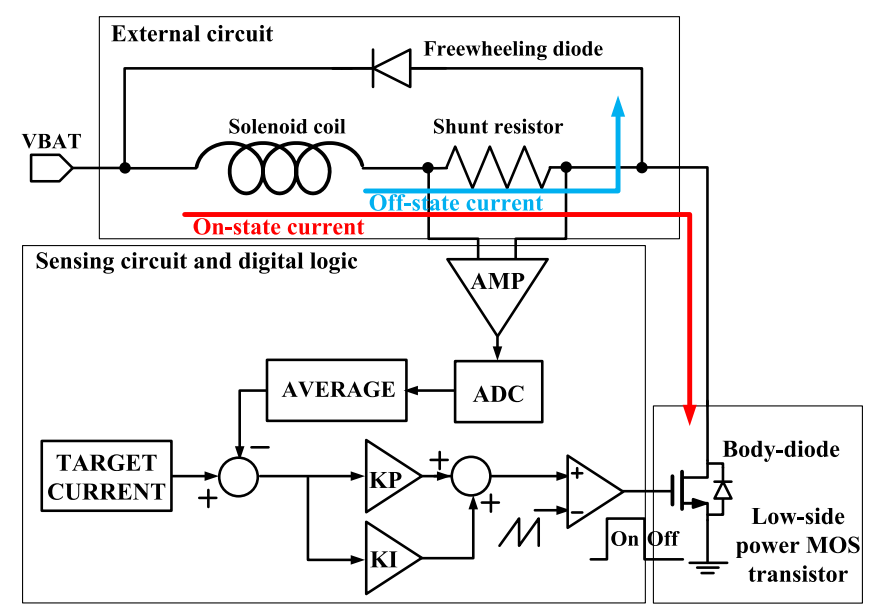

Fig. 2. Conventional low-side solenoid valve driver.

The ESC system has already been advanced enough to be mass-produced, but is still being researched to reduce the cost and size, as well as to optimize the control logic. However, it has been difficult to reduce the size and cost of the HCU in the ESC system in order to generate hundreds of bars of hydraulic pressure. Thus, more efforts have been made to reduce the weight and system cost of the ECU rather than the size and cost of the HCU.

Until recently, research for reducing the size and cost of the 12 solenoid valve drivers, which occupy the largest portion of the ECU, has been continuously carried out, and the products have finally been released $[4,5]$. The low-side constant current control pre-driver IC [4] controls the current of the solenoid coil with a shunt resistor to achieve a high current accuracy, but the shunt resistor occupies a large PCB size and increases the system cost. The low-side solenoid driver [5] achieves a high accuracy for the solenoid current with an internal shunt resistor and a freewheeling diode; however, it increases the die size and degrades thermal performances due to the off-state current flowing through an integrated freewheeling diode and a sensing resistor.

In this paper, a highly accurate solenoid valve driver with high- and low-side current sensing circuits is proposed to achieve a small size, low cost, and high current accuracy for the ESC system. In addition, various conventional solenoid valve drivers and their current sensing circuits are extensively investigated and compared with the proposed solenoid valve driver.

\section{Conventional and proposed solenoid valve drivers}

In the ECU, the MCU performs the braking operation by driving the actuators, such as a motor and solenoid valves, using sensor information at various circumstances. Generally, the HCU uses a poppet-type valve operated by a solenoid coil whose current is controlled by a PWM driving method. The ECU adjusts the current of the solenoid coil to control the magnetic and net force acting on the solenoid valve. The current flowing through the solenoid coil is sensed in various ways and applied to the analog-to-digital converter (ADC). The proportional integration (PI) controller generates the error value, which is then converted to a PWM signal to control the current of the solenoid coil [6]. 


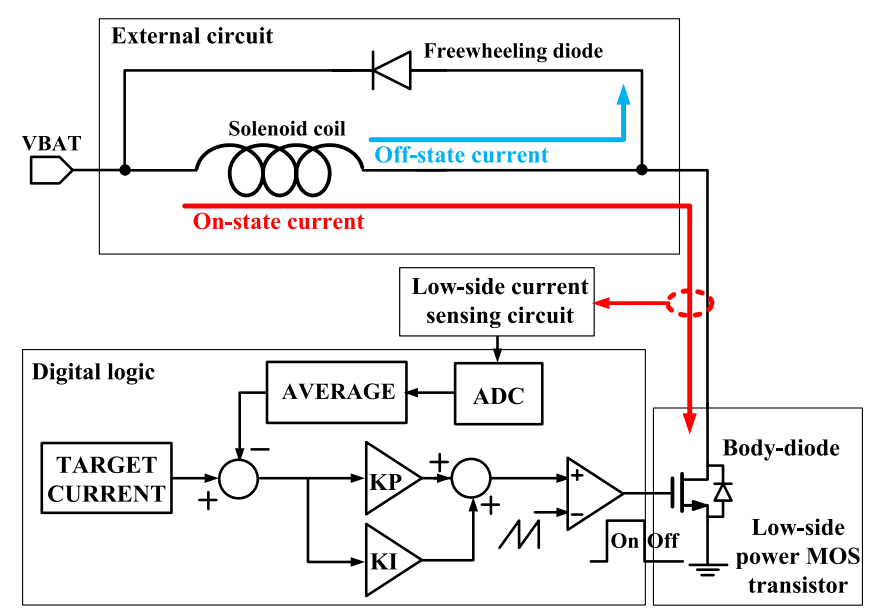

Fig. 3. Low-side solenoid valve driver without a shunt resistor.

Fig. 2 shows the conventional structure of the low-side solenoid valve driver [4], which has a shunt resistor to sense the current flowing through the solenoid coil in the on- and off-states of the low-side power MOS transistor. However, this structure employed in 8-channel PWM solenoid valve drivers suffers from a large PCB size and a high system cost due to shunt resistors occupying a large portion of the PCB area. In addition, variation in the resistance value of the shunt resistor due to the increasing temperature or deviation affects the current accuracy of the solenoid valve driver. To improve the current accuracy of this structure, temperature compensation is carried out using a lookup table; however, a temperature sensor is needed in each valve, resulting in an increase in system cost.

To improve the current accuracy of the conventional low-side solenoid valve driver and reduce the system cost, a low-side solenoid driver without a shunt resistor was introduced, as shown in Fig. 3. This low-side solenoid valve driver without a shunt resistor employs an on-state current sensing circuit to measure and control the current of the solenoid coil in the on-state of the low-side power MOS transistor instead of measuring the current flowing through the freewheeling diode in the off-state of the low-side power MOS transistor.

In this way, the ADC can be shared by other solenoid valve drivers, and thus the use of the ADC can be reduced by half, effectively reducing the die size. However, since the current of the solenoid coil has unbalanced characteristics between the on- and off-states of the low-side power MOS transistor, the low-side solenoid valve driver suffers from poor current accuracy.

Fig. 4 shows the measured data and simulation results of the solenoid coil current. The measured data shows a steep current slope right after the low-side driver is turned off or on, and then shows a gentle current slope. These unbalanced characteristics are caused by variations in the impedance value of the solenoid coil valve due to the ferromagnetic, hysteresis, and saturation characteristics of the solenoid valve core [7]. Moreover, the resistance value of the solenoid coil further varies by $70 \%$ to $100 \%$ due to the coil heating caused by high current $[8,9]$, resulting in the shape of the current ripple being affected. To analyze the characteristics of the solenoid coil current, the solenoid value was mathematically modeled and simulated using a Simulink simulation tool [7]. Thus, the simulation result is 


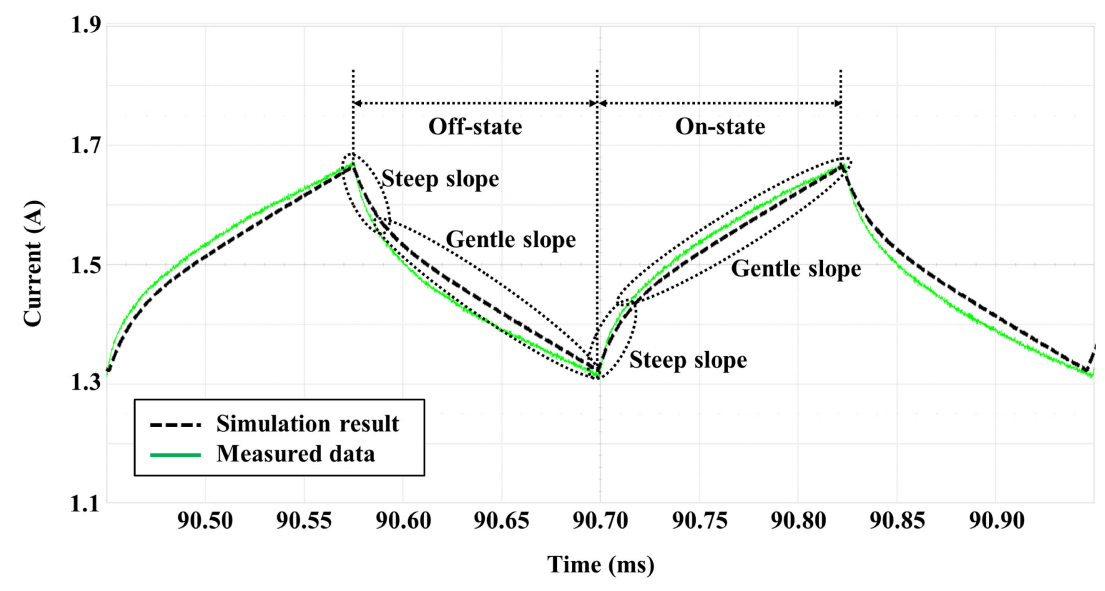

Fig. 4. Measured data and simulation result of the solenoid coil currents

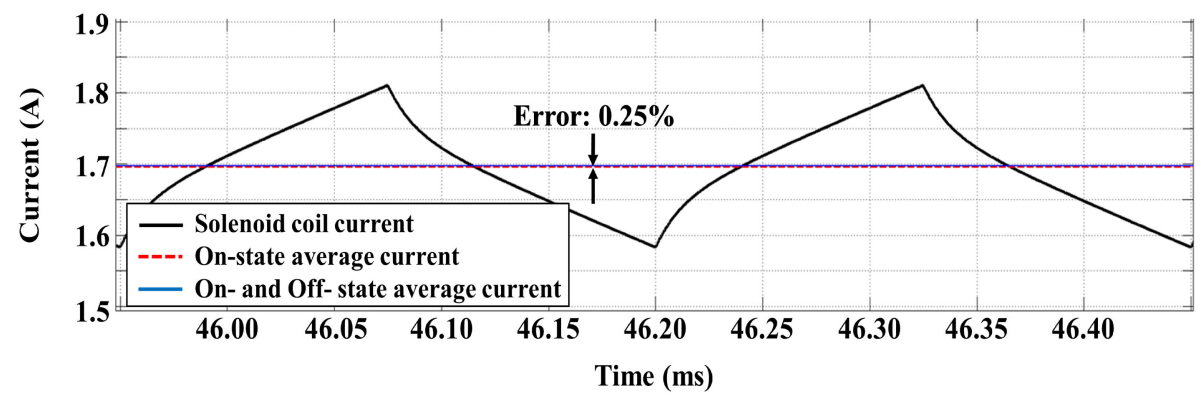

(a)

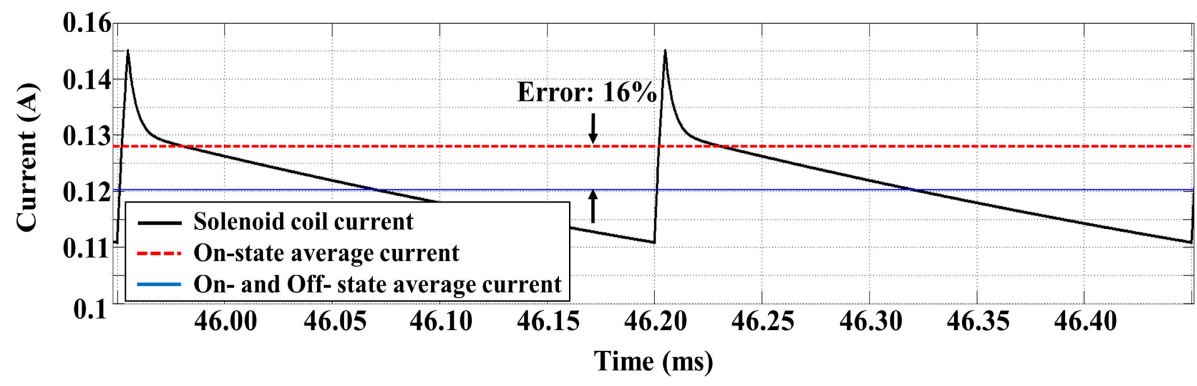

(b)

Fig. 5. Average currents in the on-state only and in the on- and offstates when the target currents are (a) $1.7 \mathrm{~A}$ and (b) $0.12 \mathrm{~A}$.

obtained using a real solenoid coil with the valve, showing that it fits well with the measured data, as shown in Fig. 4.

Fig. 5 shows the simulation results of the average currents of the solenoid valve driver in the on-state only and in the on- and off-states. The simulated errors in the average current between the on-state only and the on- and off-states are $0.25 \%$ and $16 \%$ at target currents of $1.7 \mathrm{~A}$ and $0.12 \mathrm{~A}$, respectively, indicating that the error in the average current increases as the target current decreases.

These errors occur because the solenoid valve driver operates in the short duration of the on-state of the low-side power MOS transistor to satisfy the required low target current. As the duration of the off-state becomes longer than that of the on-state, the error in the average current increases further due to unbalanced characteristics. The duration of the on-state $\left(T_{o n}\right)$ is given by 


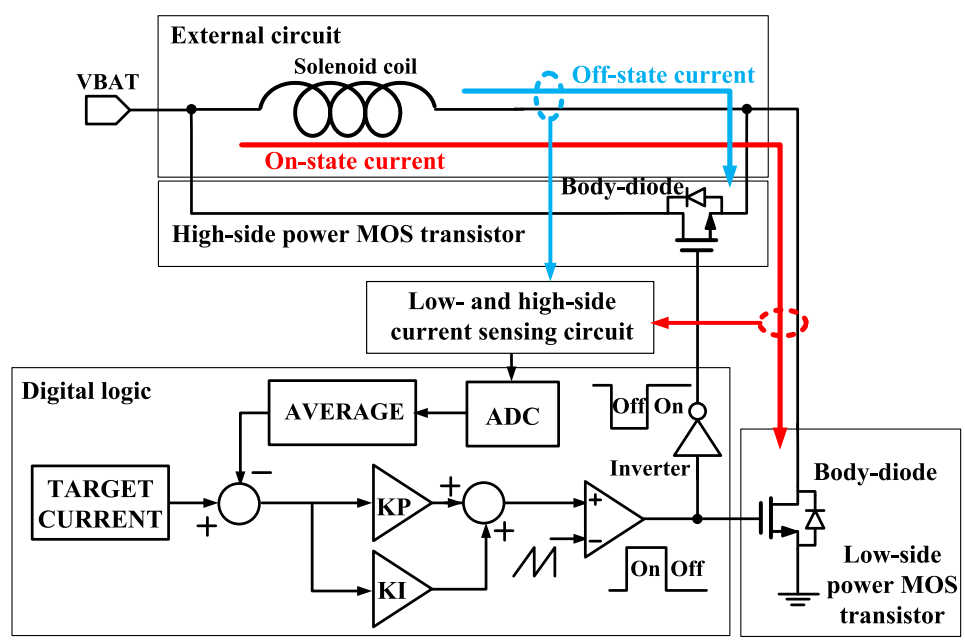

Fig. 6. Proposed solenoid valve driver.

$$
T_{\text {on }}=\frac{R_{d s, \text { on }}+R_{L}}{V_{B A T} f_{P W M}} \times I_{T A R G E T}
$$

where $I_{\text {TARGET }}$ is the target current of the solenoid valve driver, $V_{B A T}$ is the battery voltage, $f_{P W M}$ is the PWM frequency, $R_{d s, o n}$ is the on-resistance of the low-side power MOS transistor, and $\mathrm{R}_{\mathrm{L}}$ is the resistance of the solenoid coil. Therefore, the worst case condition occurs when the solenoid valve driver operates in the minimum $T_{o n}$, i.e., high $V_{B A T}$, high $f_{P W M}$, low $R_{d s, o n}$, low $R_{L}$, and low $I_{T A R G E T}$.

The accuracy at a low target current can be mitigated more or less by the compensation and calibration of the system; however, it is difficult to achieve a precise accuracy due to the impedance variation caused by the increasing temperature of the coil. Thus, a current sensing scheme is proposed to achieve a high current accuracy by sensing the current of the solenoid coil in the on- and off-states of the low-side power MOS transistor.

Fig. 6 shows the proposed solenoid valve driver, which consists of the proposed high- and low-side current sensing circuits, a digital logic, low- and high-side power MOS transistors, and an external circuit including a solenoid coil. Unlike the conventional low-side solenoid valve driver, a high-side power MOS transistor is employed in the proposed solenoid valve driver instead of a freewheeling diode.

In the proposed solenoid valve driver, since the high-side power MOS transistor has a body-diode, its power losses caused by the switching and conduction can respectively be expressed as

$$
\begin{aligned}
P_{M O S, S W}= & \frac{1}{2} \times I_{\text {TARGET }} \times V_{D S} \times\left(t_{o f f}+t_{o n}\right) \times f_{P W M} \\
& +\frac{1}{2}\left(C_{I S S} \times V_{G S}{ }^{2} \times f_{P W M}\right)+\frac{1}{2}\left(C_{O S S} \times V_{D S}{ }^{2} \times f_{P W M}\right), \\
& P_{M O S, C O N}=R_{d s, \text { on }} \times D_{O N, D U T Y} \times I_{T A R G E T}{ }^{2},
\end{aligned}
$$

where $D_{O N, D U T Y}$ is a PWM on-duty cycle, $V_{D S}$ is a drain-source voltage of the highside power MOS transistor, $t_{\text {on }}$ and $t_{\text {off }}$ are the turn-on and turn-off switching times, respectively, $C_{I S S}$ and $C_{O S S}$ are the input and output capacitances of the high-side power MOS transistor, respectively, and $V_{G S}$ is a gate-source voltage. Eq. (2) represents the switching loss caused by the body-diode, input capacitor $\left(C_{I S S}\right)$, and 
output capacitor $\left(C_{O S S}\right)$. Eq. (3) represents the conduction loss caused by $R_{d s, o n}$. Since the off-state current flows through the body-diode before the high-side power MOS transistor is turned on, $V_{D S}$ becomes almost zero according to zero voltage switching (ZVS) [10]. Therefore, the switching loss of the high-side power MOS transistor becomes negligibly small, thus the first term in Eq. (2) is considered to be almost zero, and the second and third terms dominate the switching loss.

In the conventional solenoid valve driver, the power loss of the freewheeling diode is given [11] by

$$
\begin{gathered}
P_{D I O D E, C O N}=V_{T 0} \times I_{F(A V)}+R_{D I O D E} \times I_{F(R M S)}{ }^{2}, \\
I_{F(A V)}=D_{O N, D U T Y} \times I_{T A R G E T}, \\
I_{F(R M S)}=\sqrt{D_{O N, D U T Y}} \times I_{T A R G E T},
\end{gathered}
$$

where $V_{T 0}$ is a diode threshold voltage, $I_{F(A V)}$ is an average forward current of the freewheeling diode, $R_{D I O D E}$ is a resistance of the freewheeling diode, and $I_{F(R M S)}$ is an RMS forward current of the freewheeling diode.

Using the electrical parameters in Table I, and Eqs. (2), (3), and (4), the total power losses of the high-side power MOS transistor and the freewheeling diode are calculated to be $0.6329 \mathrm{~W}$ and $0.7321 \mathrm{~W}$, respectively, indicating that the high-side power MOS transistor has lower power loss (thus, higher power efficiency) than the freewheeling diode.

Table I. Electrical parameters for high-side power MOS transistor and freewheeling diode.

\begin{tabular}{|c|c|c|}
\hline Component & $\begin{array}{c}\text { High-side Power } \\
\text { MOS Transistor }\end{array}$ & $\begin{array}{c}\text { Freewheeling Diode } \\
\text { (STPS3150U [12]) }\end{array}$ \\
\hline$I_{T A R G E T}$ & $2.25 \mathrm{~A}$ & $2.25 \mathrm{~A}$ \\
\hline$R_{d s, o n}$ & $0.25 \Omega$ & - \\
\hline$f_{P W M}$ & $4 \mathrm{kHz}$ & $4 \mathrm{kHz}$ \\
\hline$C_{I S S}$ & $500 \mathrm{pF}$ & - \\
\hline$C_{O S S}$ & $250 \mathrm{pF}$ & - \\
\hline$R_{D I O D E}$ & - & $0.027 \Omega$ \\
\hline$V_{G S}$ & $10 \mathrm{~V}$ & - \\
\hline$V_{D S}$ & $0.675 \mathrm{~V}$ & - \\
\hline$V_{T 0}$ & - & $0.59 \mathrm{~V}$ \\
\hline$D_{O N, D U T Y}$ & 0.5 & 0.5 \\
\hline
\end{tabular}

In addition, this structure compensates for the error due to the unbalanced characteristics shown in Fig. 5 by sensing the current of the solenoid coil in the offstate of the low-side power MOS transistor. Thus, the current accuracy of the solenoid coil is improved, and moreover, the PCB size of the proposed solenoid valve driver can be reduced by eliminating the necessity of the use of discrete components used in the conventional solenoid valve driver.

\section{Circuit implementation}

Fig. 7 shows the proposed high- and low-side current sensing circuits with the solenoid coil, which are implemented using a matched MOS transistor with a much 
smaller aspect ratio than the power MOS transistor [13]. The error amplifiers in the low- and high-side current sensing circuits sense the currents flowing through the low- and high-side power MOS transistors $\left(\mathrm{M}_{\mathrm{L} 1}\right.$ and $\left.\mathrm{M}_{\mathrm{H} 1}\right)$, respectively, by using the drain voltage when each power stage is turned on.

In the on-state, transistors $\mathrm{M}_{\mathrm{L} 1}-\mathrm{M}_{\mathrm{L} 6}$ are turned on, where $\mathrm{M}_{\mathrm{L} 1}, \mathrm{M}_{\mathrm{L} 2}$, and $\mathrm{M}_{\mathrm{L} 4}$ operate in the deep triode region and $\mathrm{M}_{\mathrm{L} 3}, \mathrm{M}_{\mathrm{L} 5}$, and $\mathrm{M}_{\mathrm{L} 6}$ operate in the saturation region, and the low-side error amplifier makes the voltages at nodes $V_{A}$ and $V_{B}$ equal. Thus, the on-state current $\mathrm{I}_{\mathrm{LS}}$ is mirrored to $\mathrm{M}_{\mathrm{L} 5}$ and $\mathrm{M}_{\mathrm{L} 6}$, and the mirrored current flowing through $\mathrm{M}_{\mathrm{L} 6}$ is then converted to a voltage using a resistor, RSENSE $\mathrm{LS}$.

In the off-state, transistors $\mathrm{M}_{\mathrm{H} 1}-\mathrm{M}_{\mathrm{H} 4}$ are turned on while having the same voltage at nodes $\mathrm{V}_{\mathrm{A}}$ and $\mathrm{V}_{\mathrm{C}}$, and the off-state current $\mathrm{I}_{\mathrm{HS}}$ flowing through $\mathrm{M}_{\mathrm{H} 1}$ is sensed by a high-side error amplifier. The mirrored current flowing through $\mathrm{M}_{\mathrm{H} 3}$ is then converted to a voltage using a resistor, RSENSE $E_{\mathrm{HS}}$. To operate transistors $\mathrm{M}_{\mathrm{H} 1}, \mathrm{M}_{\mathrm{H} 2}$, and $\mathrm{M}_{\mathrm{H} 4}$ in the deep triode region, a charge pump is required.

Fig. 8 shows the simulation results of the proposed high- and low-side current sensing circuits. The solenoid current ( $\mathrm{I}_{\mathrm{HS}}$ plus $\mathrm{I}_{\mathrm{LS}}$ ) reaches a target value of $820 \mathrm{~mA}$ by complementarily increasing $\mathrm{I}_{\mathrm{HS}}$ and $\mathrm{I}_{\mathrm{LS}}$ using the PWM input signals of s1 and s2 for the low- and high-side power MOS transistors, respectively.

Since the off-state current flows through the high-side power MOS transistor, the proposed sensing circuit structure has more Joule heating than other structures of the conventional solenoid valve drivers. In addition, since these conventional structures use an external freewheeling diode, effective thermal dissipation can be achieved using a dedicated exposed pad of the external freewheeling diode package. Therefore, thermal dissipation in the proposed solenoid valve driver would be a critical issue to be considered in designing an IC including 12 channel valve drivers.

To achieve effective thermal dissipation at the IC level, $R_{d s, o n}$ of the power MOS transistor should be lowered, which can be effectively done by increasing the size of the power MOS transistor. However, since the total die size increases even

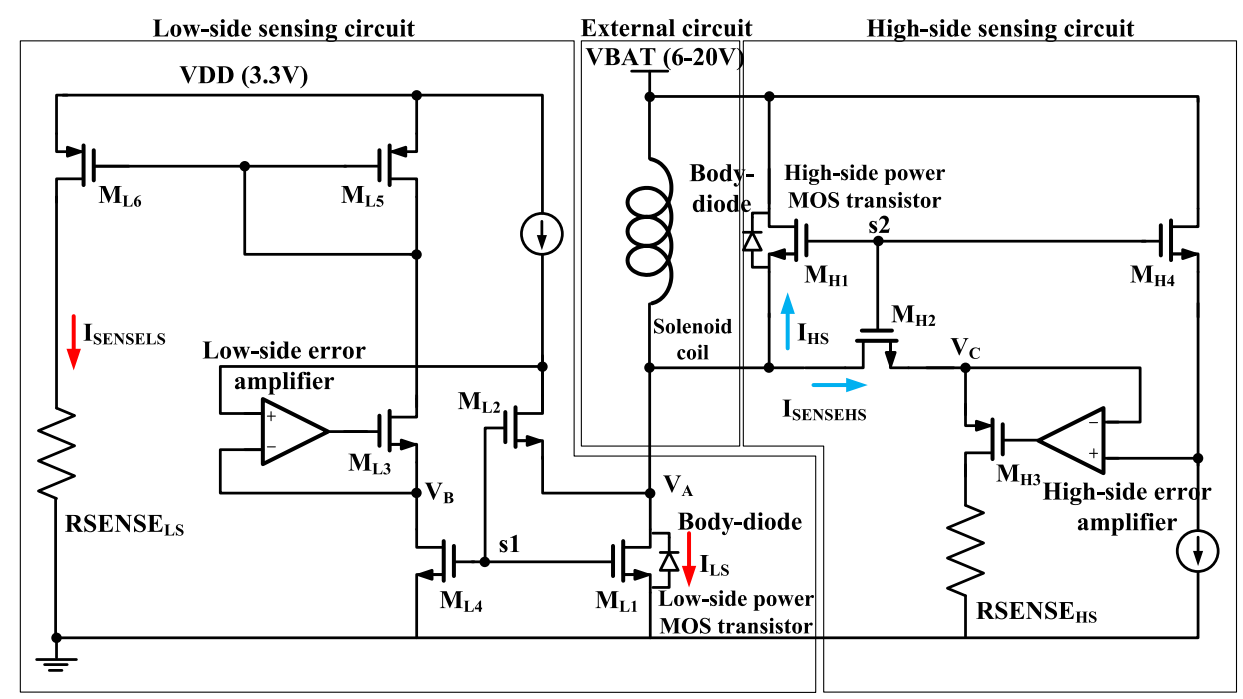

Fig. 7. Proposed high- and low-side current sensing circuits with the solenoid coil. 


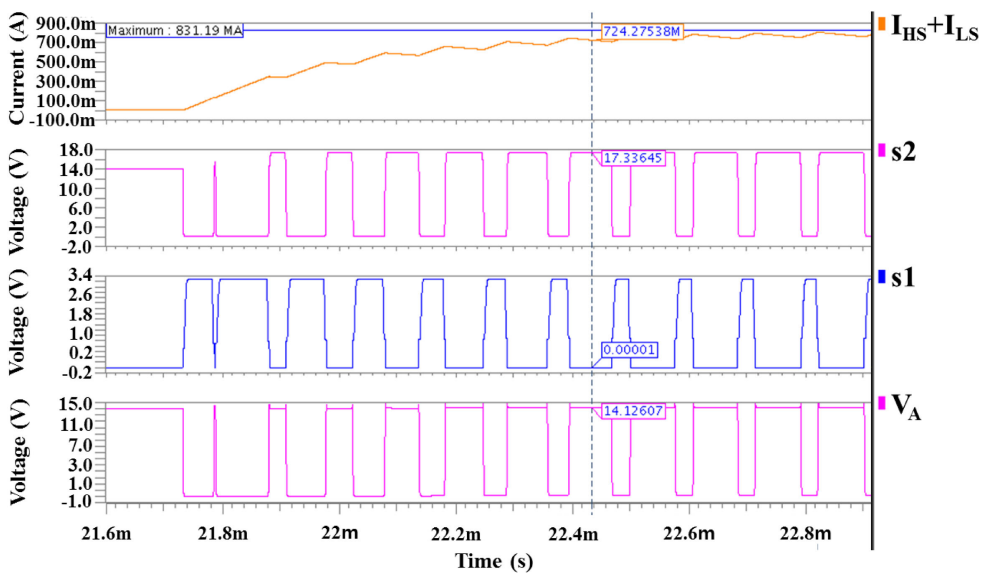

Fig. 8. Simulation results of the proposed high- and low-side current sensing circuits with the solenoid coil.

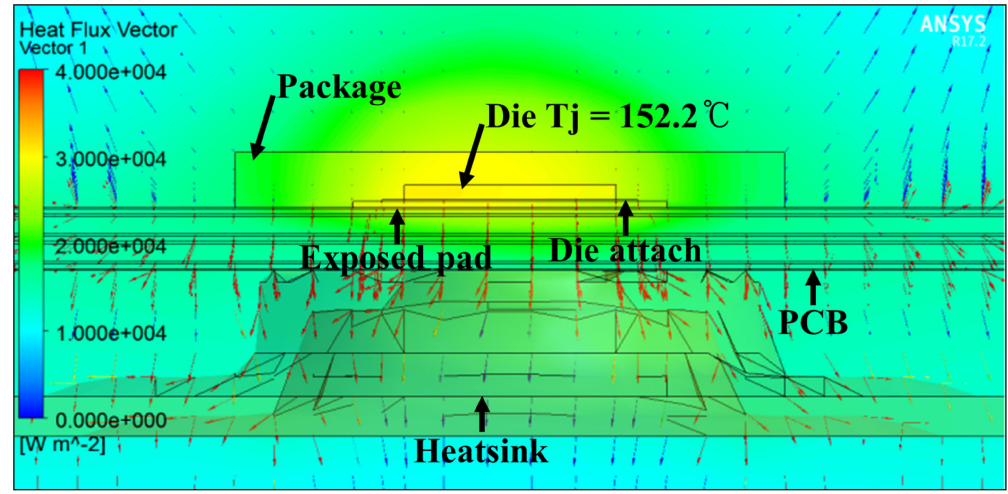

Fig. 9. Thermal simulation results.

with a small increase in the power MOS transistor size, the system cost will increase. Therefore, the method for $R_{d s, o n}$ optimization should be considered as follows.

Once the design of the solenoid valve is completed, the operating points for cut-in and cut-out and the maximum operating points of the solenoid valves are determined. This is closely related to the number of turns of the coil for increasing magnetic force, resulting in increasing the cost of coils. A solenoid coil with a large number of turns has a high magnetic force even at a low current, whereas a solenoid coil with a small number of turns requires a high current for a high magnetic force. Therefore, the number of coil turns, the solenoid coil current, and the system cost should be adjusted with respect to each other to protect the IC from damage due to the high temperature. After the operating points and the solenoid current are determined, thermal simulation is performed through the mission profile. The worst case pattern of the ESC system is shown in Fig. 9, which shows a die temperature of $152.2^{\circ} \mathrm{C}$ at an ambient temperature of $125^{\circ} \mathrm{C}$.

\section{Measurements}

Fig. 10 shows a photomicrograph of the proposed solenoid valve driver, which was fabricated using a $0.11 \mu \mathrm{m}$ BCDMOS process. 


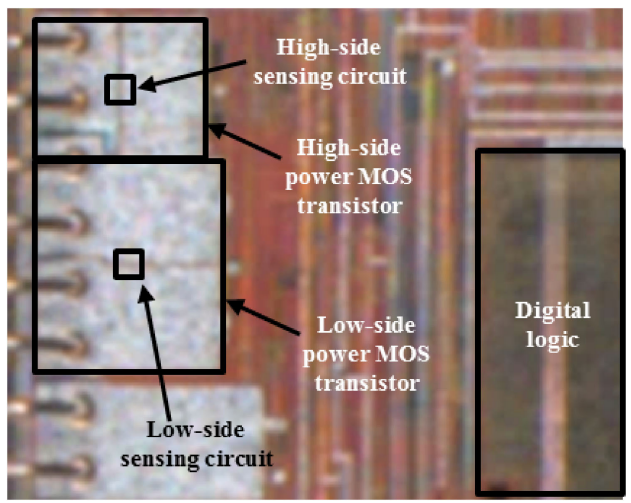

Fig. 10. Photomicrograph of the proposed solenoid valve driver.

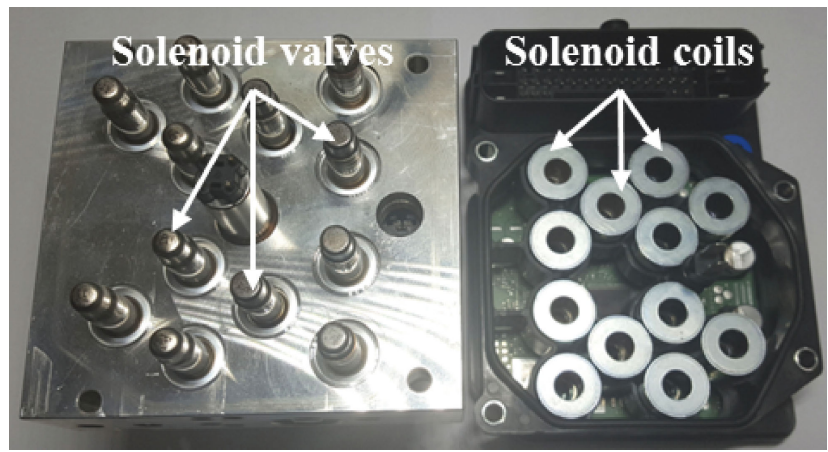

Fig. 11. Solenoid valves and coils.

The fabricated solenoid valve driver was tested using an HCU with the solenoid coils and valves shown in Fig. 11 to verify the performance of the proposed solenoid valve driver. The measurement was carried out with battery voltages ranging from $6 \mathrm{~V}$ to $20 \mathrm{~V}$ and temperatures ranging from $-40^{\circ} \mathrm{C}$ to $140^{\circ} \mathrm{C}$ at a PWM frequency of $4 \mathrm{kHz}$.

Fig. 12 shows the measured output voltage and current of the solenoid valve driver when the target currents are $260 \mathrm{~mA}, 460 \mathrm{~mA}, 1.5 \mathrm{~A}$, and $2.25 \mathrm{~A}$. The measurement results show that the duration of the on-state of the output voltage of the solenoid valve driver varies as the target average current changes, indicating that the output of the proposed solenoid valve driver is properly regulated at different target currents.

The average current accuracy of the solenoid coil controlled by the solenoid valve driver was measured under various conditions using an oscilloscope with a current probe when the solenoid coil current reaches steady state, as shown in Fig. 13.

The measurement results show that the solenoid valve driver accurately operates and the error between the target current and the inductor current is less than $\pm 4 \%$ in the entire current range from $0 \mathrm{~A}$ to $2.25 \mathrm{~A}$ except when the battery voltage is $6 \mathrm{~V}$. Since the average current cannot reach a target current at the low battery voltage of $6 \mathrm{~V}$, the measurement cannot be carried out at a target current of 1.6 A or above. The current errors are mainly due to the mismatch in size between the power MOS transistors in the proposed sensing circuit. 


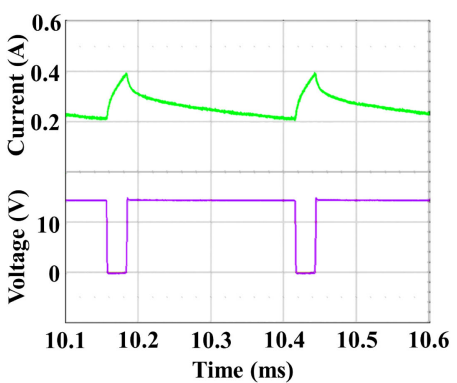

(a)

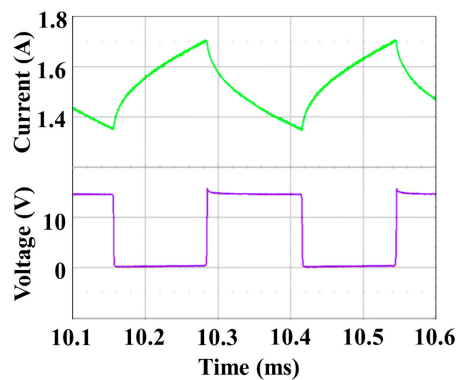

(c)

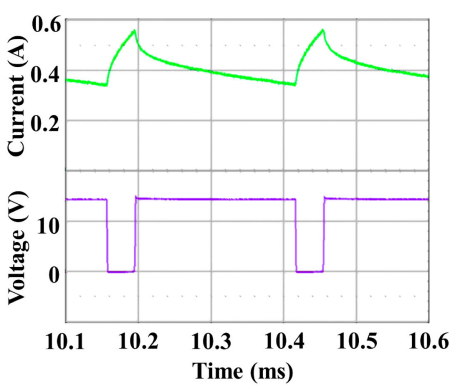

(b)

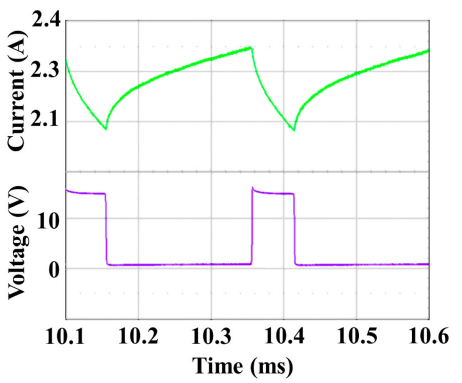

(d)

Fig. 12. Measured output voltage and current of the solenoid valve driver at steady state when the target average current is (a) $260 \mathrm{~mA}$, (b) $460 \mathrm{~mA}$, (c) $1.5 \mathrm{~A}$, and (d) $2.25 \mathrm{~A}$.

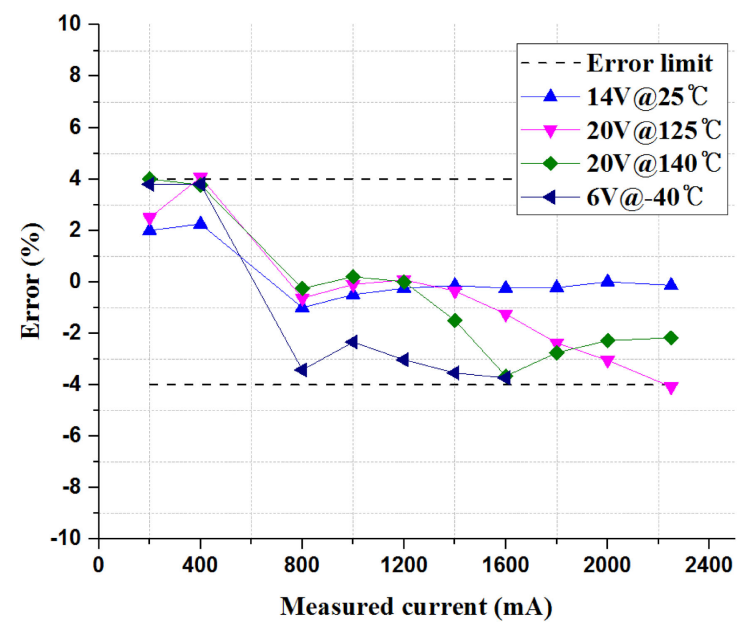

Fig. 13. Measured average current accuracy of the solenoid coil controlled by the solenoid valve driver under various conditions.

Fig. 14 shows the measurement results of the current of the solenoid valve driver in the transient state from $1.2 \mathrm{~s}$ to $3.2 \mathrm{~s}$ with a time step of $0.2 \mathrm{~s}$. When the target current varies by $225 \mathrm{~mA}$ per time step, the current of the solenoid valve driver is settled within $300 \mu \mathrm{s}$, as shown in the zoomed-in area, and thus the solenoid coil and valve can operate sufficiently within a few milliseconds.

Table II shows the performance comparison of the proposed solenoid valve driver with previous works. The proposed solenoid valve driver with the high- and low-side sensing circuits achieved an average current accuracy of $\pm 4 \%$ under various conditions and a lower system cost compared to previous works. Moreover, 


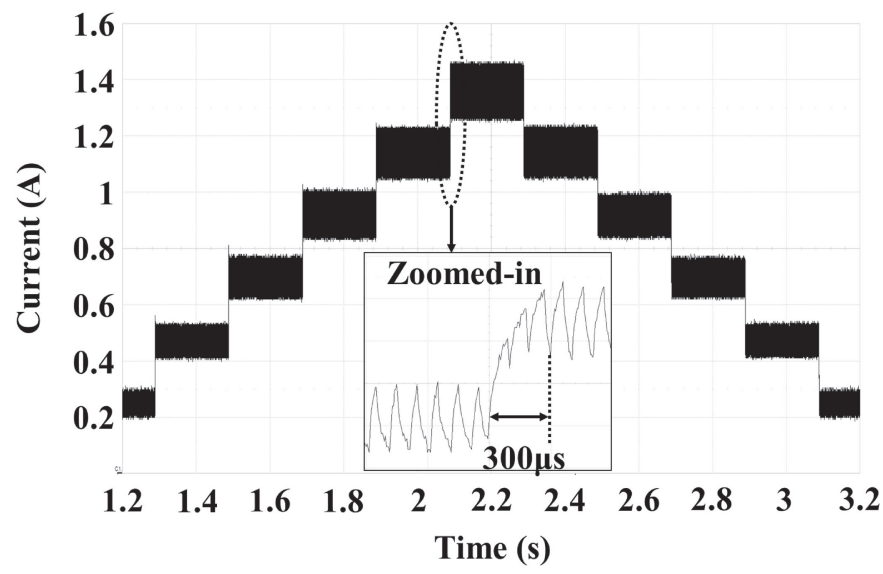

Fig. 14. The measured current of the solenoid valve driver in the transient state.

the PCB size of the ECU was reduced by eliminating the necessity of the use of discrete components, a shunt resistor and a freewheeling diode, used in the conventional solenoid valve driver.

Table II. Performance comparison with previous works.

\begin{tabular}{|l|l|l|l|l|}
\hline & TLE8242 [4] & L9352B [5] & Previous work & Proposed IC \\
\hline Structure & $\begin{array}{l}\text { Low-side } \\
\text { with external } \\
\text { shunt }\end{array}$ & $\begin{array}{l}\text { Low-side } \\
\text { with internal } \\
\text { shunt }\end{array}$ & $\begin{array}{l}\text { Low-side } \\
\text { without shunt } \\
\text { resistor }\end{array}$ & $\begin{array}{l}\text { Low-side and } \\
\text { high-side }\end{array}$ \\
\hline $\begin{array}{l}\text { Current } \\
\text { accuracy }\end{array}$ & $\pm 2 \%$ & $\pm 6 \%$ & $\pm 6 \%$ & $\pm 4 \%$ \\
\hline $\begin{array}{l}\text { Operating } \\
\text { range }\end{array}$ & $0-1.5 \mathrm{~A}$ & $0-2.25 \mathrm{~A}$ & $0-2.25 \mathrm{~A}$ & $0-2.25 \mathrm{~A}$ \\
\hline $\begin{array}{l}\text { Operating } \\
\text { voltage }\end{array}$ & $5.5-42 \mathrm{~V}$ & $4.8-18 \mathrm{~V}$ & $6-20 \mathrm{~V}$ & $6-20 \mathrm{~V}$ \\
\hline $\begin{array}{l}\text { Operating } \\
\text { temperature }\end{array}$ & $-40-125^{\circ} \mathrm{C}$ & $-40-125^{\circ} \mathrm{C}$ & $-40-125^{\circ} \mathrm{C}$ & $-40-125^{\circ} \mathrm{C}$ \\
\hline Die size & Small & Large & Small & Medium \\
\hline PCB size & Large & Small & Medium & Small \\
\hline System cost & High & Medium & Medium & Low \\
\hline
\end{tabular}

\section{Conclusion}

In this paper, a solenoid valve driver with high- and low-side current sensing circuits is proposed to achieve a high current accuracy by sensing the current of the solenoid coil alternately in the on- and off-states of the power MOS transistor. In addition, the proposed valve driver is implemented to sense the current of the solenoid coil without a shunt resistor, thereby reducing the system cost. The measurement results show that the solenoid valve driver properly controls the current of the solenoid coil under various conditions. Using the proposed high-side current sensing circuit, the solenoid valve driver not only reduces the system cost and PCB size, but also achieves an average current accuracy of $\pm 4 \%$. Therefore, the proposed solenoid valve driver is suitable for automobile brake systems. 